\title{
Environment Control Method in Solid Waste Dry Fermentation Garage based on Global Variable Prediction Model
}

\author{
P.J. WANG \& Y.S.Chen ${ }^{*} \&$ D.W.Zhu\& R.R.Li\& H.L.Qu \\ Nanjing Research Institute for Agricultural Mechanization Ministry of Agriculture, Nanjing, China
}

\begin{abstract}
The thesis put forward a new global variable prediction model whose theories, structure of modeling and operation were discussed. Applying BP neural network in solid waste dry fermentation garage environment control, the reliability and stability of the new control method was proved.

KEYWORD: Solid Waste Dry Fermentation Garage; Environment Control; Neural Network; Global Variable
\end{abstract}

\section{INTRODUCTION}

Solid waste dry fermentation garage's intelligent control system is controlled by the computer. According to the most appropriate environment with degradation of solid waste, automation technology, modern information technology and bio-technology combine to create a suitable environment for the degradation of solid waste and achieve low energy consumption and efficient processing of large-scale. Designing an intelligent control system has extremely significance[1-3].

For the first solid waste dry fermentation garage, temperature, gas production rate, $\mathrm{PH}$ value and ORP (Oxidation-Reduction Potential) of artificial monitoring, administrators regularly to the edge of the garage observes database, calculating gas production through gas meter observation, and then decide whether to warming, spray. In this way arbitrary large, the situation is difficult to precisely control, in the case of labor-intensive and inefficient. Now most dry fermentation garage have control systems, but in these systems each independently controlled actuators are usually based on the set and measured values . It must to be a professor to operate the actuators. At the same time, each actuator workplace settings independently of each other, making adjustments to work bad coordinated, easily lead the system to shock and overshoot, and the control system operates in a passive state, only when the climate in the garage changed, can not be based on future circumstances make early response.

To overcome these problems, it must be added to the intelligent control system to achieve the overall control of the global system[4]. Through the use of environmental control method in garage based on global variable prediction model, the environment inside and outside the garage, how much gas production and $\mathrm{PH}$ value, ORP as a whole system of research and analysis. The variables collected as input values, using the predicted values obtained for each controller to predict the future state of the environment of the garage, the situation timely dry fermentation garage environment to predict imminent, according to the predicted value, early adjust fermentation garage environment has greatly improved the quality of dry fermentation garage control.

\section{SOLID WASTE DRY FERMENTATION GARAGE OF INTELLIGENT CHARACTERISTICS AND REQUIREMENTS}

\subsection{Solid waste dry fermentation garage characteristics of intelligent control system}

Solid waste dry fermentation garage intelligent control system is mainly based on the temperature of the external environment, fermentation garage-based expert system and the basic parameters set by some of the control measures to regulate the temperature inside the garage, ventilation, $\mathrm{PH}$ value of other environmental factors and timely spraying marsh liquid, creating a suitable solid waste fermentation garage suitable environment(The environment is a press fermentation require different materials to optimize the development of co-ordination). According to the needs of different fermentation period of solid waste, that testing standards developed dry fermentation garage environment, through real-time monitoring, the measured parameters are automatically adjusted each control device status after relatively dry fermentation garage 
to enable compliance with the environmental factors established by the requirements.

\subsection{Solid waste dry fermentation garage control system requirements}

Solid waste dry fermentation garage intelligent control system uses artificial intelligence model to generate the control program, so that all actuators common coordination to achieve optimal control. Intelligent control is required to achieve the necessary basis of the following:

1) Control model's exactness.Fermentation to a certain period of solid waste fermentation garage, one of the garage environment will affect the regulation of solid waste fermentation, on the other hand due to transpiration garage materials sink and create new environmental factors affect the garage, thereby generating a feedback mechanism in the conventional dry fermentation environment control system does not consider the effect of this feedback mechanism. Also exist among the dry fermentation garage environment, strong couplings and the elements, certain elements of change will have an impact on other elements. Therefore, intelligent control systems need to create accurate models, achieve regulatory approach which can save resources and improve productivity.

2) Detection of large amounts of data. Accurate modeling need to collect large amounts of data (including time and space), the data collected by the sensors are to provide support for modeling. Solid waste fermentation achieve intelligent control model garage, not only need to consider the value of the internal fermentation treasurer parameters such as temperature, $\mathrm{PH}$ value, ORP, etc., also need to consider the external environment of the garage fermentation parameters, as well as local real-time weather conditions and prediction of short-term weather conditions.

\section{SOLID WASTE REPOSITORY OVERALL STRUCTURE OF DRY FERMENTATION}

Using two small-scale experiments solid waste dry fermentation garage, a single database volume $75 \mathrm{~m}^{3}$, garage roof insulation plastic, dry fermentation garage is divided into 10 regional monitoring the thermal region shown in Figure 1.

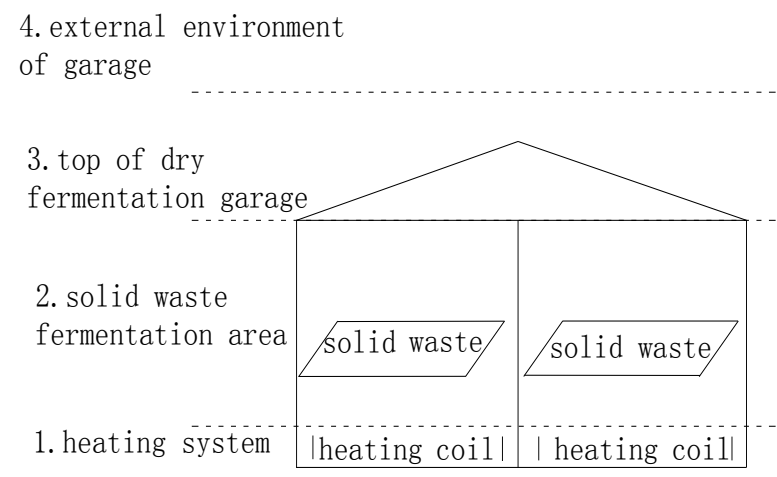

Figure 1. Experiment temperature heat area schematic diagram

Taking into account the coupling of dry fermented garage of the parameters, the dry fermentation garage is divided into four regions. Each locale is a collection of data related to the sensor, the sensor data acquisition and category type as shown in Table 1 (experiment considering only the temperature, $\mathrm{PH}$ value, ORP, gas production , but COD and other factors are not discussed. )

Table 1. The regional of dry fermentation garage data acquisition's type and quantity.

\begin{tabular}{|c|c|c|c|c|}
\hline area & Data acquisition category & Number of Temperature sensors & Number of PH meter & Number of ORP meter \\
\hline 1 & Heating system & 1 & 0 & 0 \\
\hline 2 & Solid waste fermentation area & 10 & 1 & 1 \\
\hline 3 & Top of dry fermentation garage & 1 & 0 & 0 \\
\hline 4 & External environment of garage & 1 & 0 & 0 \\
\hline
\end{tabular}

\section{SOLID WASTE DRY FERMENTATION GARAGE CONTROL PROGRAM}

In the dry fermentation garage, the main factors affecting the degradation and solid waste management costs are inside temperature, $\mathrm{PH}$ value, such as these two parameters can accurately predict and control in this forecast, we can achieve the best control effect, the control program shown in Figure2.

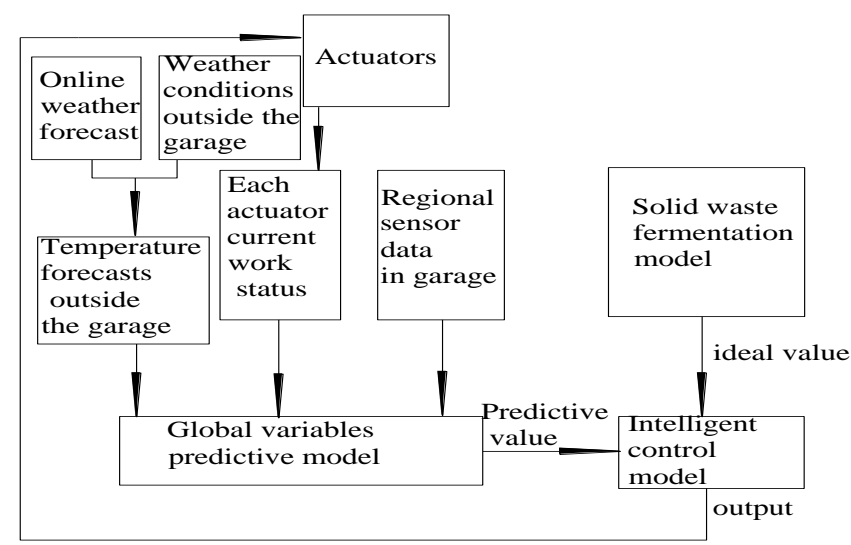

Figure 2. Control structure 


\subsection{Global variables predictive model}

\subsubsection{Dry fermentation garage external temperature prediction module}

This dry fermentation garage temperature prediction module using an outdoor temperature sensor and often a combination of weather forecasts predicting dry fermentation garage external climate models, from the perspective of the prediction accuracy using analog methods, the use of external sensors to collect data, combined with the weather conduct trend forecasting analogy, dry fermentation garage to changing external weather conditions to make appropriate judgments to external short-term dry fermentation garage climate (after a few hours) to make predictions. However, when the data is not accurate weather forecasts or network failure and data corruption, you must use an approximate data to replace the data. Alternative data can use the following formula (empirical formula) to obtain approximated.

$$
T_{R}=\alpha_{0}+\alpha_{1} T_{L}+\alpha_{2} R_{L}+\alpha_{3} R_{L}^{2}
$$

Where $T_{R}=$ local approximation temperature; $T_{L}=$ actual temperature; $R_{L}=$ local light intensity values $; \alpha_{i}=$ weighting factor; $\alpha_{i}$ using linear regression data fitting, conducted periodically adjusted according to the actual situation, which ranges between 0 and 1 .

\subsubsection{Global variables Predictive models}

Total of forty-six global variables, including sixteen future temperature values, two light intensity values, two ORP values, six execution units working state values, an online weather forecast provided by the neural network model achieved. Global variables predictive model inputs divided into three parts.

1) Each zone temperature, ORP and light intensity measurements collected by the various sensors inside the dry fermentation garage.

2) Dry fermentation garage external weather forecast values by the online weather, combined with dry fermentation garage external environment variables to provide predictive value.

3) Actuators of the program's current configuration (including insulation, ventilation, heating, spray, etc.), which means that each actuator working condition, as predicted global variable reference.

\subsection{Dry fermentation garage intelligent control model}

Intelligent control model on the basis of the input, predicted the output state values. The internal environment is used to adjust the dry fermentation garage. There is two parts in the model:
1) Setting the value of the solid waste to the top of the degradation phase model (temperature, $\mathrm{PH}$ ), i.e. the desired value. Degradation models include solid waste degradation various stages required optimum temperature, $\mathrm{PH}$ and other conditions.

2) Predictive value of the global forecast model (temperature, $\mathrm{PH}$ value). The model has six inputs, respectively, solid waste degradation phase optimum temperature, $\mathrm{PH}$ value, and global variables prediction model in the dry fermentation forecasts the future temperature and $\mathrm{PH}$ value. Six outputs are namely six signal of actuator control. The function of the model uses PID control to achieve.

\subsection{BP neural network model}

BP neural network from with the input layer, hidden layer and output layer, hidden layer can be extended to multiple layers. This process is repeated alternately, and ultimately the global minimum error in the network tends given to complete all the learning process. The structure shown in Figure 3:

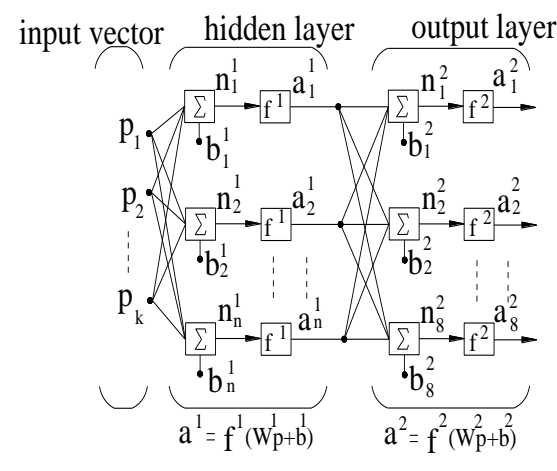

Figure 3. BP neural network structure

In this study the structure of BP neural network, take the three-layer structure, namely an input layer, a hidden layer and an output layer [5], in which the number of neurons in the input layer is 46, corresponding to 46 global variables; output layer neuron number is 3 , the corresponding three predictive value (temperature, $\mathrm{PH}$ value, ORP value); hidden layer obtained from the following empirical formula, rounded to the nearest integer, after a total of 25 neurons.

$$
N_{h}=\frac{N_{i}+N_{0}}{2}
$$

Where $N_{h}=$ hidden layer neurons; $N_{i}=$ input layer neurons; $N_{0}=$ output layer neurons.

If $X_{i}=$ input mode; $Y_{k}=$ output mode vectors. The hidden layer to the output node is

$$
O_{j}=f\left(\sum_{i=1}^{n} X_{i} \times W_{i j}\right)
$$

The output of the output layer is 


$$
Y_{k}=f\left(\sum_{j=1}^{n} O_{j} \times T_{j k}\right)
$$

Which excitation function is consecutive values of Sigmoid function between 0 and 1.

$$
f(x)=\frac{1}{1+e^{-x}}
$$

Deviation is calculated using

$$
E=\frac{1}{2} \sum\left(t_{i}-O_{i}\right)^{2}
$$

Where $t_{i}=$ desired value of the No. $i$ output samples; $O_{i}=$ actual output value of the No. $i$ node. Initial weights using a random number generator, the range $[-0.5,0.5]$, using the formula weight matrix correction.

$$
W_{i j}(t+1)=W_{i j}(t)-\eta \frac{\partial E}{\partial W_{i j}(t)}
$$

Where $\eta=$ efficiency of learning, in this study, $\eta=0.62$; training samples selected environment variable between December 20, 2013 to May 26, 2014 inside the dry fermentation garage.

Before using BP neural network, there must be training, the training procedure is as follows

1) Select the No. $k$ set of samples, $\left(X_{k}, Y_{k}\right)$, $k=1,2, \ldots \mathrm{m}$, entering $X_{k}$ into network;

2) Using a random number generator to generate the initial weights;

3) Using Equation (6) to calculate the deviation. If the deviation result is $E<\varepsilon(\varepsilon=0.001)$ then quit the count;
4) Calculate $\frac{\partial E_{k}}{\partial W}$
5) Calculate $\frac{\partial E}{\partial W}=\sum_{k=1}^{m} \frac{\partial E_{k}}{\partial W}$

6) Using formula

7) Correction weights

After training, the weights of the neural network has been determined, you can use the network to ask for the next period of time within the dry fermentation garage environment variables to predict.

\section{EXPERIMENTAL RESULTS AND ANALYSIS}

For global variables predictive models for the relevant test (due to the solid waste dry fermentation garage does not have a good complement of optical devices, so there is no compensation for lighting control, only the temperature, $\mathrm{PH}$ value control), dry fermentation garage materials is straw mixed cow dung, pilot programs designed as follows:
1) Select at $07: 00$ on January 5th, 2014 to at 06:00 on January 6, 2014, a total of $24 \mathrm{~h}$ as a test stage, the use of value-based forecasting model output, the implementation of solid waste on the environment dry fermentation garage control, come to control the outcome;

2) Select the February 17, 2014 to March 1, 2014 total 2 weeks as a test phase, using the same method for the implementation of solid waste dry fermentation garage control, findings of the trial;

3) Respectively control the use of predictive models and conventional PID control simulation comparison, comparing the merits of the two methods.

Figure shows the temperature and $\mathrm{PH}$ test date changes Figure 4. At this point dung mixed straw just in the dry fermentation dry fermentation garage of materials, optimum temperature range of 25 $35^{\circ} \mathrm{C}$, optimum $\mathrm{PH}$ range of 7.4 8.0.

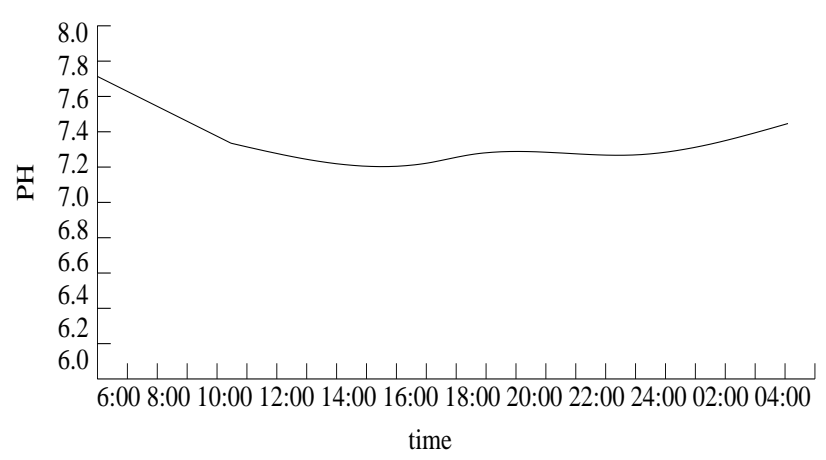

a. $\mathrm{PH}$ value change

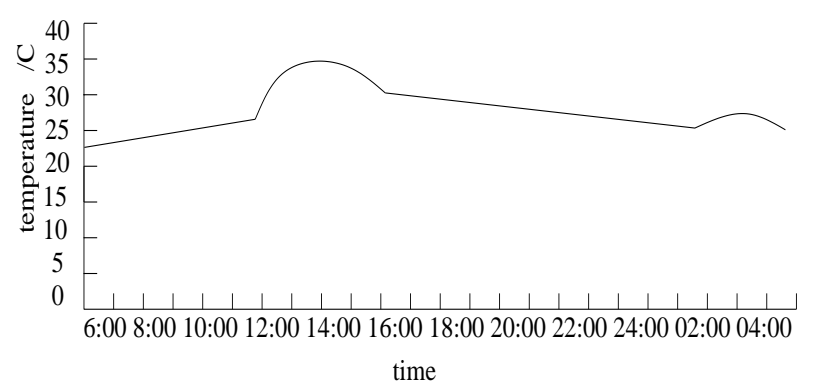

b. Temperature change

Figure 4. PH value and temperature in 24 hours of dry fermentation period

As can be seen from Figure 4, in order to predict the solid waste dry fermentation garage environment control predictive value provided by the base model, in the day to ensure that the garage temperature between $25-35^{\circ} \mathrm{C}$, garage $\mathrm{PH}$ is between 7.4-7.8.

During two weeks, the average daytime temperature and averages $\mathrm{PH}$ value is shown in Figure 5. As can be seen from Figure 5, the whole of cattle manure straw can be guaranteed in the fermentation chamber temperature between $25-35^{\circ} \mathrm{C}$, $\mathrm{PH}$ is between 7.4-7.8. Whether a single day or stage control, the control system can guarantee the environmental degradation of solid waste in the best state, to illustrate the effectiveness of this method. 


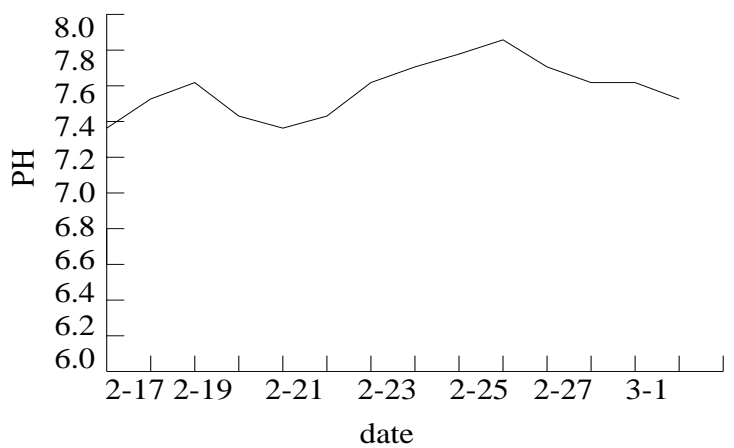

a. Average daily $\mathrm{PH}$

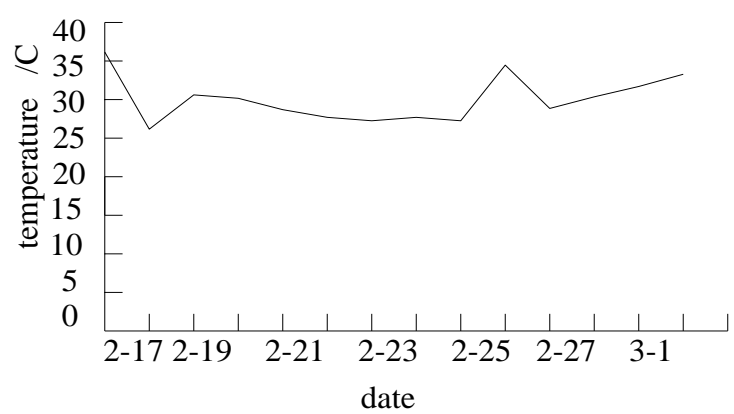

b. Average daily temperature

Figure 5. The average daily mean temperature and PH change Figure in two weeks of dry fermentation period.

Prediction model using global variables to control and use the comparison of PID control simulation, shown in Figure 6. As can be seen from the results, the use of global variables predictive model control method, the inside temperature and $\mathrm{PH}$ changes than conventional PID control changes to the gentle and more effective forecasting model explain the method to avoid the large inertia of the entire control system and bring big lag and overshoot, system stability has been greatly enhanced.

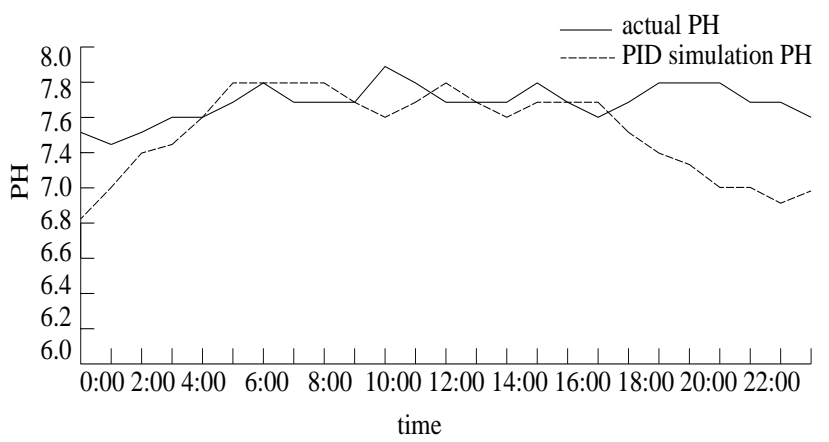

a.PH

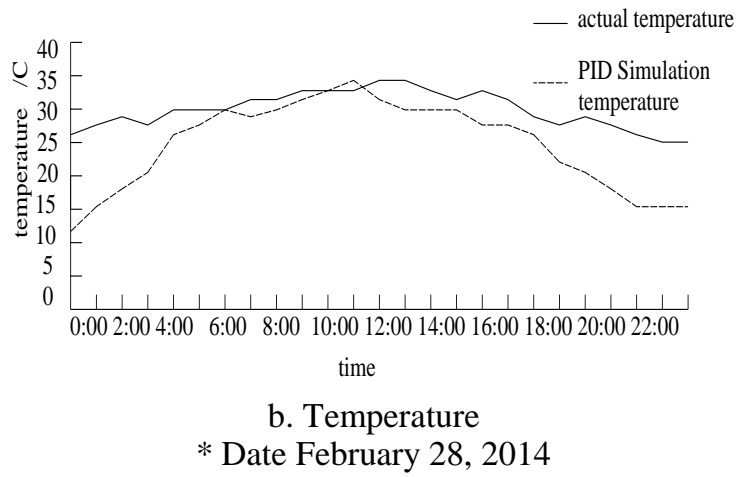

Figure 6. Two control methods of $\mathrm{PH}$ and temperature variations comparison chart.

\section{CONCLUSION}

The thesis suggests the model of a global variable prediction that can be used in a solid waste dry fermentation garage environment control. Based on BP neural network control method, the research derives the formulas of neural network and of calculating the temperature outside the dry fermentation garage. The reliability and stability of the design is proved by the actual temperature and $\mathrm{PH}$ value.

\section{ACKNOWLEDGEMENTS}

Project supported by the Jiangsu Province Science and Technology support Plan (Grant NO.BE2012775)

\section{REFERENCES}

[1] Chen Guangqing, Xun Aiqin, Xu Kebao. 2010 The monitor and control system for greenhouse based on PLC and configuration software, Journal of Anhui Agriculture ,38(34): 19827-19828

[2] Ma Wanzheng, Ma Wanmin.2011.The Current Situation and Trend of Intelligent Environment Control Technology in Greenhouse. Northern Horticulture.11(23): 179-180.

[3] Mao Hanping.2007.Situation and Development of Facilities of Agriculture. Agriculture Equipment \& Technology, 7(5): 6-10.

[4] Qu Yi, Ning Duo, Lai Zhanchi, et al.2011. Neural networks based on PID control for greenhouse temperature. Transactions of the Chinese Society of Agricultural182Engineering, 27(2):307-311.

[5] Dayhoff J E, Deleo J M. 2001Artificial neural Cancer, 91(8): 1615-1634. 